\title{
CLINICAL PROFILE OF PATIENTS ADMITTED WITH ACUTE KIDNEY INJURY IN INTENSIVE CARE UNIT
}

\author{
Manjunath Kulkarni ${ }^{1}$, Anto Gnana Delasalle2, Arunachalam ${ }^{3}$ \\ ${ }^{1}$ Associate Professor, Department of Nephrology, Father Muller Medical College Hospital, Mangalore. \\ ${ }^{2}$ Senior Resident, Department of Medicine, Father Muller Medical College Hospital, Mangalore. \\ 3 Professor, Department of Medicine, Father Muller Medical College Hospital, Mangalore.
}

\begin{abstract}
BACKGROUND
ABSTRACT

Acute Kidney Injury (AKI) in intensive care unit (ICU) is very common. This study looked at the clinical profile of patients admitted in ICU with AKI.
\end{abstract}

\section{MATERIALS AND METHODS}

Prospective observational study was done in an ICU of a tertiary care centre in Mangalore. Patients admitted to the ICU who were 18 years of age and had diagnosis of AKI by KDIGO criteria were included in the study. Data was collected and analysed.

\section{RESULTS}

Total number of patients included in the study was 100 . Diabetes mellitus was the most common comorbidity seen in $38 \%$ cases followed by Hypertension in $27 \%$. The duration of ICU stay ranged from 4 days to 12 days. Sepsis was the most common cause of AKI (51\%). Forty-five percent of patients required haemodialysis.

\section{CONCLUSION}

Sepsis was the most common aetiological factor responsible for AKI. Diabetes mellitus was found to be the most common co-morbid illness associated with development of acute kidney injury. Respiratory tract infection was found to be the most common diagnosis at admission among those with AKI. Most AKI in ICU have oliguria. Acute tubular necrosis was found to be the most common pathophysiological process responsible for AKI. Forty-five percent of ICU-AKI patients require RRT. SOFA score of $\geq 9$ and increasing severity of AKI were associated with increased mortality and also poor rate of recovery from acute kidney injury and increased mortality.

\section{KEWORDS}

Acute Kidney Injury, Intensive Care Unit.

HOW TO CITE THIS ARTICLE: Kulkarni M, Delasalle AG, Arunachalam. Clinical profile of patients admitted with acute kidney injury in intensive care unit. J. Evolution Med. Dent. Sci. 2017;6(95):6927-6930, DOI: 10.14260/jemds/2017/1503

\section{BACKGROUND}

Acute Kidney Injury (AKI) in Intensive Care Unit (ICU) is associated with high mortality and morbidity. Knowledge about the aetiology and risk factors leading to AKI will help devise appropriate interventions at the right time and improve the overall outcome of patients with AKI. There is a relative paucity of data regarding clinical profile of AKI in ICU from Indian subcontinent. Hence, this study was planned to look at the clinical profile of patients admitted in ICU with AKI and ascertain aetiology, risk factors leading to AKI. We also looked at relationship between severity of AKI and outcomes in terms of need for dialysis, recovery and mortality.

\section{MATERIALS AND METHODS}

This prospective observational study was conducted in an ICU of Father Muller Medical College Hospital, a tertiary care centre in Mangalore from October 2014 to March 2016. Patients admitted to the ICU who were 18 years of age and had diagnosis of AKI by KDIGO criteria were included in the study. Staging of AKI was based on KDIGO guidelines.(1)

'Financial or Other Competing Interest': None.

Submission 03-11-2017, Peer Review 05-12-2017,

Acceptance 11-12-2017, Published 23-12-2017.

Corresponding Author:

Manjunath Kulkarni,

Associate Professor,

Department of Nephrology,

Father Muller Medical College, Mangalore.

E-mail:drmjkulkarni@gmail.com

DOI: $10.14260 /$ jemds $/ 2017 / 1503$
Informed written consent was obtained from all patients. Standard demographic data (age, gender and date of admission) was obtained from all participants enrolled. Clinical and physiological data collected included the diagnosis at admission, presence of co-morbidities (hypertension, diabetes mellitus and coronary artery disease) and the requirement of mechanical ventilation. Physiological data included the Glasgow Coma Scale (GCS), $\mathrm{PaO}_{2} / \mathrm{FiO}_{2}$ ratio, electrolytes, bilirubin and blood counts. The data on renal function included urine microscopy, blood urea, serum creatinine and urine output. The investigations were repeated when deemed warranted by the treating physician. Severity of illness on admission and during ICU stay was assessed using the SOFA score. Duration of ICU stay was noted. Patients were followed up till being transferred out from ICU.

The need, frequency and modality of dialysis when administered was noted. All data was recorded on a predefined format. The probable causes for AKI were classified as follows-

- Nephrotoxic Drugs: If the patient had received a drug with known nephrotoxic potential for a minimum period of two days prior to the defined increase in serum creatinine concentration.

- $\quad$ Sepsis: When two or more of the following were present as a result of systemic infection.

1. Temperature $>38.0$ or $<36.0^{\circ} \mathrm{C}$;

2. Heart rate $>90 / \mathrm{min}$;

3. Respiratory rate $>20 / \mathrm{min}$ or $\mathrm{pCO}_{2}<32 \mathrm{~mm}$; and

4. White blood cell count $>12,000 / \mathrm{mm}^{3}$ or $<4000$ $\mathrm{mm}^{3}$ or $>10 \%$ band forms. 
- Hypotension: If there was overt/ orthostatic hypotension (BP $<80 \mathrm{mmHg}$ ), because of purely cardiovascular cause.

- Volume Loss: If there was obvious cause of volume depletion along with loss of skin turgor with decrease of more than $5 \%$ of body weight.

- $\quad$ Sepsis and Drugs: If there were sepsis as well as use of drugs, which are potentially nephrotoxic.

- Volume Loss and Sepsis: When there was volume loss as well as septicaemia.

- Sepsis-Drugs-Hypotension: If there was septicaemia, use of drugs which are potentially nephrotoxic as well as hypotension because of cardiovascular cause.

- Envenomation and Poisoning: AKI due to snake bite and poisoning by organophosphorus compounds etc.

\section{Definition of AKI Recovery}

1. Oliguric Patients: Increase in urine output and progressive decline in serum creatinine after initial attainment of stable values or dialysis independent at the time of transfer out from ICU.

2. Non-Oliguric patients: Progressive decline in serum creatinine after initial attainment of stable values or dialysis independent at the time of transfer out from ICU.

Data was analysed using SPSS Ver 20 and results were interpreted as frequencies, proportions, means and standard deviation by using chi-square and Fisher's exact tests.

\section{RESULTS}

\section{Demographic Data}

Total number of patients included in the study was 100, of which 57 were males and 43 were females. Mean age of the patients included in the study was $54.9 \pm 14.55$ yrs. Seventy two percent patients had comorbidities. Diabetes mellitus was the most common comorbidity seen in $38 \%$ cases followed by Hypertension in $27 \%$. The duration of ICU stay ranged from 4 days to 12 days with an average of 6.9 days.

Respiratory tract infection was seen in $25 \%$ of the study population. The other sites of infection were genitourinary tract (12\%) and gastrointestinal tract (4\%).

\section{COMORBIDITIES}

\begin{tabular}{|c|c|}
\hline & Number of Cases \\
\hline $\begin{array}{c}\text { Chronic Liver Disease with Portal } \\
\text { Hypertension }\end{array}$ & 5 \\
\hline Chronic Obstructive Pulmonary Disease & 14 \\
\hline Diabetes Mellitus & 38 \\
\hline Hypertension & 27 \\
\hline Ischaemic Heart Disease & 12 \\
\hline Seizure Disorder & 3 \\
\hline \multicolumn{2}{|c|}{$\begin{array}{c}\text { adable 1. Comorbidities of Patients } \\
\text { admitted in ICU with AKI }\end{array}$} \\
\hline
\end{tabular}

\begin{tabular}{|c|c|}
\hline Respiratory Tract Infections & 25 \\
\hline Urinary Tract Infections & 12 \\
\hline Gastrointestinal Tract Infections & 4 \\
\hline Ischaemic Heart Disease & 5 \\
\hline Poisoning and Envenomation & 6 \\
\hline Leptospirosis & 8 \\
\hline Dengue & 1 \\
\hline Malaria & 5 \\
\hline Chronic Liver Disease with Portal Hypertension & 1 \\
\hline Cerebrovascular Accident & 7 \\
\hline Diabetic Ketoacidosis & 4 \\
\hline
\end{tabular}

\begin{tabular}{|c|c|}
\hline Acute Pancreatitis & 4 \\
\hline Metabolic Encephalopathy & 8 \\
\hline $\begin{array}{c}\text { Chronic Obstructive Pulmonary Disease } \\
\text { with Type 2 Respiratory Failure }\end{array}$ & 4 \\
\hline Skin and Soft Tissue Infections & 2 \\
\hline Meningoencephalitis & 1 \\
\hline Seizure Disorder & 2 \\
\hline Neuroleptic Malignant Syndrome & 1 \\
\hline Table 2. Primary Diagnosis of Patients admitted with AKI \\
\hline
\end{tabular}

\begin{tabular}{|c|c|}
\hline & $\begin{array}{c}\text { Aetiology of Acute } \\
\text { Kidney Injury }\end{array}$ \\
\hline Sepsis & 51 \\
\hline Sepsis and Drugs & 22 \\
\hline Envenomation and Poisoning & 6 \\
\hline Nephrotoxic Drugs & 6 \\
\hline Sepsis and Volume Loss & 6 \\
\hline Sepsis, Drugs and Hypotension & 5 \\
\hline Hypotension & 2 \\
\hline Volume Loss & 2 \\
\hline \multicolumn{2}{|c|}{ Table 3. Aetiology of AKI in ICU } \\
\hline
\end{tabular}

Sepsis was found to be the major cause for Acute Kidney Injury with almost $51 \%$ of AKI being attributed to it. Twentytwo percent of those with AKI were found to be in Stage 1, 44\% in Stage 2 and 34\% in Stage 3. Oliguria was found to be present in $70 \%$ of those with AKI. Acute tubular necrosis was present in $58 \%$ of patients.

Forty-five percent of patients required haemodialysis. The rest were managed conservatively. Twenty-six percent of patients in our study required Mechanical Ventilatory support. Among those who required mechanical ventilator, 16 were males and 10 were females. Fifty-seven percent of patients recovered from AKI. Inotropes were required in $37 \%$ of patients. The mortality rate was $26 \%$.

\section{Laboratory Data}

The mean highest serum creatinine was $2.9 \mathrm{mg} \%$. Patients with highest serum creatinine $\leq 2.5$ were found to have lower rate of need for haemodialysis, whereas those with highest serum creatinine $>3.5$ were found to have higher rate of need for haemodialysis $(\mathrm{p}<0.001)$. Patients with highest serum creatinine $\leq 2.5$ were found to have higher rates of recovery, whereas those with Highest Serum Creatinine $>3.5$ were found to have lower rates of recovery $(p<0.001)$. Patients with highest serum creatinine $\leq 2.5$ were found to have lower rate of mortality, whereas those with highest serum creatinine $>3.5$ were found to have higher rates of mortality ( $p<0.001)$. Sequential Organ Failure Assessment (SOFA) Score was calculated for every patient and the mean values were tabulated. Patients with a SOFA score of $\geq 9$ were found to have higher rate of mortality $(\mathrm{p}<0.001)$ and also a higher rate of need for haemodialysis $(\mathrm{p}<0.001)$. Patients in higher stages of AKI were found to have higher mortality rates compared to those in lower stages $(\mathrm{p}<0.001)$.

\section{DISCUSSION}

The study included 100 subjects, of which $57 \%$ were males and $43 \%$ were females. In the study conducted by Prakash et al, $56.5 \%$ were males and $43.5 \%$ were females.(2) In a similar study conducted by Wijewickrama et al, $61.5 \%$ were males and $38.5 \%$ were females.(3) The mean age of patients included in the study was 54.9 yrs. with a standard deviation of 14.55 . 
Prakash et al in his study found the mean age to be $44.9 \pm 17.1$ yrs.(2) In the study done by Wijewickrama et al, mean age was found to be 47.8 yrs. with a SD of 19.4 yrs.(3) Wen $Y$ et al in his study found that the mean age was 62 yrs. with a SD of 12 yrs.(4)

Seventy-two percent of subjects had one or more comorbidities. Diabetes mellitus was found to be the most common co-morbid illness associated with AKI (38\%) followed by hypertension (27\%), COPD (14\%) and ischaemic heart disease $(12 \%)$.

In the study conducted by Prakash et al, $52.70 \%$ of patients had other co-morbid illness with hypertension being the most common $(34.7 \%)$ followed by coronary artery disease $(30.4 \%)$ and diabetes mellitus (28.3\%).(2) A higher percentage of patients with AKI had comorbidities in our study as compared to Prakash et al. This may be due to changing profile of admissions to hospital or increased incidence of noncommunicable diseases in the general population.

The duration of ICU stay ranged from a minimum of 4 days to maximum of 12 days with an average of $6.9 \pm 1.7$ days. In the study conducted by Prakash et al, mean duration of ICU stay was found to be $6.41 \pm 4.81$ days. ( $\left.^{2}\right)$

Oliguria was found in $70 \%$ of those with AKI. This was similar to the findings of Prakash et al, where oliguria was seen in $65.2 \%$ of patients with AKI.

Most causes of AKI are multifactorial and exact underlying pathology is difficult to discern. Acute Tubular Necrosis was found to be one of the major pathophysiological cause for AKI (58\%).

Twenty-six percent of patients with AKI were on invasive mechanical ventilatory support. In the study by Prakash et al $67.4 \%$ were on mechanical ventilatory support,(2) whereas $75 \%$ were on mechanical ventilatory support in the study done by Wijewickrama et al.(3) Wen $\mathrm{Y}$ et al in his study found that $68 \%$ of those with AKI were on mechanical ventilatory support.(4) The reason for this difference is not clear.

Thirty-seven percent of patients with AKI required inotropic support. Mataloun et al found that $47.72 \%$ of those with AKI were on inotropic support,(5) whereas in the study done by Wen Y et al 30.8\% were on inotropic support.(4)

Of the 100 patients studied, $45 \%$ of patients required haemodialysis for acute kidney injury. Prakash et al found that in his study $54.34 \%$ of patients required renal replacement therapy, similarly in the study done by Wijewickrama et al $55.38 \%$ of patients with AKI required haemodialysis.(3,2) These findings were similar to that in our study.

Fifty-seven percent of patients recovered from acute kidney injury. In the study done by Prakash et al, recovery from AKI was seen in $42.7 \%$ of patients.(2)

In our study, mortality was seen in $26 \%$ of patients. In the study done by Wijewickrama et al, mortality was seen in $47.7 \%$ of patients.(3) In the study done by Prakash et al, the mortality was $63 \%$.(2) The low rate of mortality in our study could be attributed to factors like the profile of patients admitted, improving standards of ICU care and also due to early initiation of corrective measures for AKI.

Respiratory tract infections were found to be the most common diagnosis at admission for patients with AKI followed by urinary tract infections, leptospirosis, metabolic encephalopathy, poisoning and envenomation being the other common causes. The study done by Mataloun et al also had similar findings. Respiratory tract infections were found to be the most common cause for admission to ICU among those with AKI.(5)

Sepsis was found to be the most common aetiological cause for acute kidney injury with almost $51 \%$ of cases being attributed only to it. This finding is similar to many studies, which have shown sepsis to be the major cause for AKI. Prakash et al in his study found sepsis to be the major cause for AKI with $69.9 \%$ of subjects.(2) In the study done by Wijewickrama et al, sepsis was the primary diagnosis for $45.4 \%$ of patients. ${ }^{(3)}$

Sepsis along with nephrotoxic drugs, volume loss and hypotension was found to be associated with almost $84 \%$ of cases of acute kidney injury. The other aetiological causes were nephrotoxic drugs and envenomation and poisoning.

Twenty-two percent were found to be in Stage 1, 44\% in Stage 2 and the remaining 34\% in Stage 3. In the study done by Wijewickrama et al, $35.38 \%$ were in Stage 1, Stage 2 comprised of $6 \%$ of patients and Stage 3 had $58.65 \%$.(3)

Higher SOFA score was associated with increased need for dialysis, lower recovery rates from AKI and increased mortality. In a study done by Wijewickrama et al those with a SOFA score of more than 9 were found to have a mortality rate of $53.1 \%$, whereas it was $27.1 \%$ in those with a SOFA score of $\leq 9$.(3) Wen $\mathrm{Y}$ et al in his study found that those with a mean SOFA score of more than 9 was associated with a mortality of $55.3 \% .(4)$

Peak serum creatinine of $>2.5 \mathrm{mg} / \mathrm{dL}$ showed a direct relationship with the need for dialysis and mortality. It also showed an inverse relationship with recovery from AKI, $(\mathrm{p}<$ 0.001).

Few studies have compared highest serum creatinine with outcomes. The study done by Mataloun et al showed increased mortality rate of $26 \%$ among those with highest serum creatinine value $\geq 3$ and $10 \%$ among those $<3$. Our study findings also confirm the same.(5)

The severity of AKI was assessed based on KDIGO staging. It was found that subjects in Stage 1 had $100 \%$ recovery as compared to those in higher stages. Twenty percent at Stage 3 , showing an inverse relationship between severity and recovery from AKI ( $\mathrm{p}$ 0.001).

Those in Stage 3 were found to have higher mortality rates $52.9 \%$ when compared to Stage 2 and Stage $1 \mathrm{AKI}$.

In the study done by Wen Y et al, those in Stage 1 were found to have mortality of $31 \%$ and those in Stage 2 had a mortality rate of $36 \%$ and those in Stage 3 with a mortality of 99\%.(4)

Wijewickrama et al in his study found that those in Stage 1 had a mortality rate of $43 \%$, subjects in Stage 2 had a mortality rate of $50 \%$ and in Stage 3 mortality rate was $55 \%$. 3 .

The findings correlated with that of our study; hence, those with increasing severity of AKI were found to have higher mortality rates.

\section{CONCLUSION}

Sepsis was the most common aetiological factor responsible for AKI. Diabetes mellitus was found to be the most common comorbid illness associated with development of acute kidney injury. Respiratory tract infection was found to be the most common diagnosis at admission among those with AKI. Most AKI in ICU have oliguria. Acute tubular necrosis was found to be the most common pathophysiological process responsible for AKI. Forty-five percent of ICU-AKI patients require RRT. 
SOFA score of $\geq 9$ and increasing severity of AKI were associated with increased mortality and also poor rate of recovery from acute kidney injury and increased mortality.

\section{REFERENCES}

[1] Kidney Disease Improving Global Outcomes (KDIGO). Acute Kidney Injury Work Group. KDIGO Clinical Practice Guideline for acute kidney injury. Kidney International Supplement 2012;2:124-38.

[2] Prakash J, Murthy AS, Vohra R, et al. Acute renal failure in the intensive care unit. J Assoc Physicians India 2006;54:784-8.
[3] Wijewickrama ES, Ratnayake GM, Wikramaratne C, et al. Incidences and clinical outcomes of acute kidney injury in ICU: a prospective observational study in Sri Lanka. BMC Research Notes 2014;7:305.

[4] Wen Y, Jiang L, Xu Y, et al. Prevalence, risk factors, clinical course and outcome of acute kidney injury in Chinese intensive care units: a prospective cohort study. Chin Med J (Engl) 2013;126(23):4409-16.

[5] Mataloun SE, Machado FR, Senna APR, et al. Incidence, risk factors and prognostic factors of acute renal failure in patients admitted in an intensive care unit. Critical Care 2003;7(Suppl 3): p. 45. 